\title{
The fracture studies of polycrystalline silicon based MEMS
}

\author{
Shantanu S. Mulay ${ }^{1}$, Gauthier Becker ${ }^{1}$, Renaud Vayrette ${ }^{2}$, Jean-Pierre Raskin ${ }^{2}$, Thomas \\ Pardoen ${ }^{2}$, Ludovic Noels ${ }^{1, *}$ \\ 1. University of Liege, Department of Aerospace and Mechanical Engineering, Computational \& \\ Multiscale Mechanics of Materials, Chemin des Chevreuils 1, B-4000 Liège, Belgium \\ ${ }^{2}$, Université Catholique de Louvain, Ecole Polytechnique de Louvain, Place du Levant 3, 1348 \\ Louvain-la-Neuve, Belgium
}

*Corresponding author. Tel:+32 43664826 E-mail address: L.Noels@ulg.ac.be

The advantages of micro-electro-mechanical system (MEMS), such as low power requirement, miniature sizes and costs reduction, has already made significant impact in many technological fields. MEMS are now widely used as accelerometers, pressure sensors, and resonators. However, the determination of mechanical properties of MEMS devices with high accuracy is still a challenging task due to their miniature size. For example, the crystal size and orientation may have a significant effect on the fracture strength of MEMS microstructure. Among several important materials used for the development of MEMS devices, polycrystalline silicon plays a key role in various applications.

Thus, an understanding of the relationship between poly-silicon manufacturing processes, and the resulting mechanical behaviour and durability of micro-fabricated structures is needed to allow rational design of MEMS devices. This work focuses on the modelling and simulation of the fracture of MEMS, made up of polycrystalline silicon, by discontinuous Galerkin (DG) formulation combined with an extrinsic cohesive law. The problem is solved as plane-stress type. All the elements are discontinuous in the DG method, and inter-element continuity is weakly enforced by having recourse to interface elements. When a fracture criterion is reached, an extrinsic cohesive law is activated on the already existing interface elements. The cracks can be initiated, merged or propagated, in this method. The fracture properties of material can be considered different along crystal interfaces and inside crystal bulk, and can account for crystal orientation. Moreover this method avoid drawbacks of usual cohesive method (no modification of the structure stiffness and no complex implementation in parallel) allowing for accurate large scale simulation. Polycrystalline silicon is an orthotropic material, i.e., it has different material properties, such as Young's modulus, Poisson's ratio, fracture strength, along different crystal lattice planes. These properties are also symmetric for a cubic crystal, i.e., the lattice planes perpendicular to each 
other have similar properties. This symmetric nature is well captured in the proposed work by a new formulation to compute effective fracture strength along any plane of interest based on the known values of the same along (1 000$),\left(\begin{array}{lll}1 & 1 & 0\end{array}\right)$, and (1 111 l lattice planes. The effect of thickness of MEMS is also included by computing the effective stress on interface elements at different vertical angles. These formulations are analyzed by sample simulation results.

The efficiency and accuracy of the method are assessed by experiments involving on-chip fracture tests, providing the fracture stress, and supplemented by the characterization of the test specimen. The characterization consists of the measurement of grain size and crystallographic orientation by electron back scattering diffraction, and the determination of crack path. An important point is the analysis of the competition between inter-granular versus trans-granular crack path with respect to the grain orientation.

[1] G. Becker, C. Geuzaine, and L. Noels. "A one Field Full Discontinuous Galerkin Method for Kirchhoff-Love Shells Applied to Fracture Mechanics." Computer Methods in Applied Mechanics \& Engineering 200, no. 45-46 (2011): 3223-3241.

[2] S. Gravier, M. Coulombier, A. Safi, N. André, A. Boe, J.-P. Raskin, J.-P., T. Pardoen, "New On-Chip Nanomechanical Testing Laboratory - Applications to Aluminum and Polysilicon Thin Films." Journal of microelectromechanical systems, 18, no. 3 (2009): 555 - 569 